\title{
A PROPERTY OF COMPACT OPERATORS
}

\author{
HERBERT KAMOWITZ
}

\begin{abstract}
In this note it is shown that if $T$ is a compact linear operator on a wide class of Banach spaces of the form $C(S)$, compact $S$, or $L^{1}(S, \Sigma, \mu)$, then $\|I+T\|$ $=1+\|T\|$. This generalizes similar theorems for the spaces $C[0,1]$ and $L^{\prime}(0,1)$.
\end{abstract}

In [1] and [2] it was shown that if $T$ is any compact linear operator on either of the Banach spaces $L^{1}(0,1)$ or $C[0,1]$, then $T$ satisfies $\|I+T\|=1+\|T\|$, where $I$ is the identity operator. In both papers the method used was to prove this for operators with finite-dimensional range, and from this deduce the theorem for arbitrary compact operators. In this note we use characterizations of compact operators on $C(S)$ or $L^{1}(S, \Sigma, \mu)$ to prove similar theorems for general Banach spaces of these forms.

\section{1. $C(S)$.}

THEOREM A. Let $S$ be a compact Hausdorff space and $C(S)$ be the supnorm Banach space of continuous complex valued functions on $S$. Then every compact linear operator $T$ on $C(S)$ satisfies $\|I+T\|=1+\|T\|$ if and only if $S$ is a perfect set, i.e. $S$ has no isolated points.

Proof. Before beginning the proof we note the following.

I. Suppose $f \in C(S)$ with $\|f\|=1$. Then two applications of Urysohn's Lemma gives that to each open set $U$ and $p \in U$ there exists $F \in C(S)$ with $\|F\|=1$, $F(p)=1$ and $F(t)=f(t)$ for $t \notin U$.

II. Let $s_{0} \in S$ and suppose $\mu$ is a measure in the dual space of $C(S)$. Since such measures are regular it follows that to each $p \in S$ and $\varepsilon>0$ there exists an open set $U$ with $p \in U$ and $|\mu(U \backslash\{p\})|<\varepsilon[3$, p. 137].

III. If $T: C(S) \rightarrow C(S)$ is a compact operator then there exists a family $\left\{d \mu_{s}\right\}_{s \in S}$ of measures on $S$ such that for each $f \in C(S)$ and $s \in S, T f(s)=\int_{S} f(t) d \mu_{s}(t)$ and further the map $s \rightarrow d \mu_{s}$ is continuous in the norm topology on $C(S)^{*}$ [3, p. 490]. As a result, if $\varepsilon>0$ and $s_{0} \in S$, there exists a neighborhood $U$ of $s_{0}$ such that $s \in U$ implies $\left\|d \mu_{s}-d \mu_{s_{0}}\right\|<\varepsilon$.

Now suppose $S$ is a compact Hausdorff space with no isolated points and let $T$ be a compact linear operator on $C(S)$. Suppose further that $T$ has the form $T f(s)=$ $\int_{S} f(t) d \mu_{s}(t)$ for $s \in S$ as described in III. Let $g \in C(S)$ with $\|g\|=1$ and

Received by the editors March 28, 1983.

1980 Mathematics Subject Classification. Primary 47B05.

(c) 1984 American Mathematical Society $0002-9939 / 84 \$ 1.00+\$ .25$ per page 
$\|T g\|=\|T\|$. Then

$$
\|T\|=\sup _{s}\left|\int_{S} g(t) d \mu_{s}(t)\right|=\left|\int_{S} g(t) d \mu_{s_{0}}(t)\right|
$$

for some $s_{0} \in S$. By multiplying $g$ by the appropriate constant of modulus 1 we may assume that $\int_{S} g(t) d \mu_{s_{0}}(t)>0$ so that $\|T\|=\int_{S} g(t) d \mu_{s_{0}}(t)$.

Let $\varepsilon>0$. Then by II and III there exists an open set $U$ such that $s_{0} \in U$, $\left|\mu_{s_{0}}\left(U \backslash\left\{s_{0}\right\}\right)\right|<\varepsilon / 4$ and if $s \in U$, then $\left\|d \mu_{s}-d \mu_{s_{0}}\right\|<\varepsilon / 2$. Since $s_{0}$ is not an isolated point of $S$, there exists $s_{1} \in U, s_{1} \neq s_{0}$, and by $I$ there exists a function $F \in C(S)$ with $F\left(s_{1}\right)=1,\|F\|=1, F\left(s_{0}\right)=g\left(s_{0}\right)$ and $F(t)=g(t)$ for $t \notin U$. Then

$$
\|I+T\| \geqslant\|(I+T) F\| \geqslant\left|(I+T) F\left(s_{1}\right)\right|=\left|F\left(s_{1}\right)+\int_{S} F(t) d \mu_{s_{1}}(t)\right| .
$$

Now

$$
\begin{aligned}
F\left(s_{1}\right)+\int_{S} F(t) d \mu_{s_{1}}(t)= & F\left(s_{1}\right)+\int_{S} F(t)\left[d \mu_{s_{1}}(t)-d \mu_{s_{0}}(t)\right] \\
& +\int_{S}[F(t)-g(t)] d \mu_{s_{0}}(t)+\int_{S} g(t) d \mu_{s_{0}}(t) .
\end{aligned}
$$

But $F\left(s_{1}\right)=1$ and $\int_{S} g(t) d \mu_{s_{0}}(t)=\|T\|$, while

$$
\left|\int_{S} F(t)\left[d \mu_{s_{1}}(t)-d \mu_{s_{0}}(t)\right]\right| \leqslant\left\|d \mu_{s_{1}}-d \mu_{s_{0}}\right\|<\frac{\varepsilon}{2}
$$

and

$\left|\int_{S}[F(t)-g(t)] d \mu_{s_{0}}(t)\right|=\left|\int_{\left.U \backslash s_{0}\right\}}[F(t)-g(t)] d \mu_{s_{0}}(t)\right|<2\left|\mu_{s_{0}}\left(U \backslash\left\{s_{0}\right\}\right)\right|<\frac{\varepsilon}{2}$.

Therefore

$$
\begin{aligned}
\|I+T\| & \geqslant\|(I+T) F\| \geqslant\left|(I+T) F\left(s_{1}\right)\right| \\
& =\left|F\left(s_{1}\right)+\int_{S} F(t) d \mu_{s_{1}}(t)\right| \geqslant 1+\|T\|-\varepsilon .
\end{aligned}
$$

This holds for all $\varepsilon>0$. Therefore $\|I+T\| \geqslant 1+\|T\|$. But $\|I+T\| \leqslant 1+\|T\|$ always. Thus if $S$ has no isolated points then $\|I+T\|=1+\|T\|$ for all compact operators $T$ on $C(S)$.

Conversely, suppose $S$ has an isolated point $s_{0}$. Define $e_{s_{0}}: S \rightarrow \mathrm{C}$ (complex numbers) by $e_{s_{0}}\left(s_{0}\right)=1$ and $e_{s_{0}}(s)=0$ for $s \neq s_{0}$. Then $e_{s_{0}} \in C(S)$. If $T$ is defined on $C(S)$ by $T f(s)=-f\left(s_{0}\right) e_{s_{0}}$ for $f \in C(S)$, then $T$ has one-dimensional range and is therefore a compact operator on $C(S)$. Further if $f \in C(S)$, then $(I+T) f(s)=$ $f(s)$ for $s \neq s_{0}$ and $(I+T) f\left(s_{0}\right)=0$. Therefore $\|I+T\|=1<1+\|T\|$. Thus if a compact set $S$ has an isolated point then there exists a compact operator $T$ on $C(S)$ with $\|I+T\|<1+\|T\|$, and the proof is complete. 
2. $L^{1}(S, \Sigma, \mu)$. Our results in this case are not as complete as for $C(S)$. Here we consider positive $\sigma$-finite measure spaces $(S, \Sigma, \mu)$ which satisfy the following additional property which we call Property (V).

Property (V). A positive $\sigma$-finite measure space $(S, \Sigma, \mu)$ is said to satisfy Property (V) if to each $s_{0} \in S$ there exists a decreasing family $\left\{E_{n}\left(s_{0}\right)\right\}$ in $\Sigma$ satisfying

(i) $s_{0} \in E_{n}\left(s_{0}\right)$,

(ii) $\mu\left(E_{n}\left(s_{0}\right)\right)>0$,

and such that if $f$ is an integrable function on $S$ with values in a Banach space, then for almost all $s_{0} \in S$,

$$
\lim _{n \rightarrow \infty} \frac{1}{\mu\left(E_{n}\left(s_{0}\right)\right)} \int_{E_{n}\left(s_{0}\right)} f(s) d \mu(s)=f\left(s_{0}\right) .
$$

The convergence is convergence in the Banach space norm. Further if $\mu\left(\left\{s_{0}\right\}\right)=0$, then $\left\{E_{n}\left(s_{0}\right)\right\}$ may be chosen to satisfy $\lim _{n \rightarrow \infty} \mu\left(E_{n}\left(s_{0}\right)\right)=0$.

Property (V) holds for a wide class of measures including Lebesgue measure on $R^{n}$, or on cubes in $R^{n}$. Indeed, it holds for arbitrary complete regular measures on these sets. Property (V) is related to the question of when certain Vitali conditions hold [3, p. 217; 4, p. 187; 5, p. 209].

We now prove a theorem similar to Theorem A for the Banach spaces $L^{1}(S, \Sigma, \mu)$ where $(S, \Sigma, \mu)$ is a positive $\sigma$-finite measure space satisfying Property $(\mathrm{V})$. We first consider the case when the measure $\mu$ has an atom.

Proposition. If $(S, \Sigma, \mu)$ is a positive $\sigma$-finite measure space and if $\mu\left(\left\{s_{0}\right\}\right) \neq 0$ for some $s_{0} \in S$, then there exists a compact linear operator $T$ on $L^{1}(S, \Sigma, \mu)$ satisfying $\|I+T\|<1+\|T\|$.

Proof. Let $s_{0} \in S$ with $\mu\left(\left\{s_{0}\right\}\right) \neq 0$ and again let $e_{s_{0}}(s)=1$ if $s=s_{0}$ and $e_{s_{0}}(s)=0$ otherwise. Then $e_{s_{0}} \in L^{\mathrm{l}}(S, \Sigma, \mu)$ and if $T f(s)=-f\left(s_{0}\right) e_{s_{0}}$ for $f \in$ $L^{\mathrm{l}}(S, \Sigma, \mu)$, then $T$ is a well-defined compact linear operator on $L^{1}(S, \Sigma, \mu)$. Since $(I+T) f(s)=0$ if $s=s_{0}$ and $(I+T) f(s)=f(s)$ for $s \neq s_{0}$, it follows that

$$
\|(I+T) f\|=\int_{S \backslash\left\{s_{0}\right\}}|f(s)| d \mu(s) \leqslant \int_{S}|f(s)| d \mu(s)=\|f\| .
$$

Hence $\|I+T\|=1<1+\|T\|$.

As a converse we have

Theorem B. Let $(S, \Sigma, \mu)$ be a positive $\sigma$-finite measure space satisfying Property (V) and suppose further that for each $s \in S,\{s\}$ is measurable and $\mu(\{s\})=0$. If $T$ is a compact linear operator on $L^{1}(S, \Sigma, \mu)$ then $\|I+T\|=1+\|T\|$.

Proof. Let $T$ be a compact operator on $L^{1}(S, \Sigma, \mu)$. Then there exists a $\mu \times \mu$ measurable function $K: S \times S \rightarrow \mathrm{C}$ such that $T f(t)=\int_{S} K(s, t) f(s) d \mu(s)$. Further,

$$
\|T\|=\operatorname{ess} \sup _{s} \int_{S}|K(s, t)| d \mu(t)
$$


and

(ii) there exists a set $E$ of measure 0 and a norm compact subset $\mathscr{K} \subset L^{1}(S, \Sigma, \mu)$ such that if $K_{s}$ is defined by $K_{s}(t) \equiv K(s, t)$ for $(s, t) \in S \times S$, then $K_{s} \in \mathcal{K}$ for all $s \notin E[3$, p. 507].

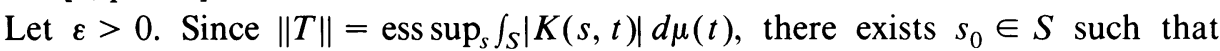
$s_{0} \notin E$ and $\int_{S}\left|K\left(s_{0}, t\right)\right| d \mu(t) \geqslant\|T\|-\varepsilon$. Also since $(S, \Sigma, \mu)$ satisfies Property (V) there exists a decreasing family of measurable sets $\left\{E_{n}\left(s_{0}\right)\right\} \equiv\left\{E_{n}\right\}$ such that $\mu\left(E_{n}\right)>0, \lim _{n \rightarrow \infty} \mu\left(E_{n}\right)=0$ and the vector valued function $s \rightarrow K_{s}$ satisfies

$$
\lim _{n \rightarrow \infty} \frac{1}{\mu\left(E_{n}\right)} \int_{E_{n}} K_{s} d \mu(s)=K_{s_{0}}
$$

in norm.

Suppose such $s_{0} \in S$ and $\left\{E_{n}\right\}$ are chosen. Then for each positive integer $n$ let $g_{n}(s)=1 / \mu\left(E_{n}\right)$ if $s \in E_{n}$ and $g_{n}(s)=0$ otherwise. Clearly, $g_{n} \in L^{1}(S, \Sigma, \mu)$ and $\|g\|=1$. Further

$$
\left\|(I+T) g_{n}\right\|=\int_{S}\left|g_{n}(t)+\int_{S} K(s, t) g_{n}(s) d \mu(s)\right| d \mu(t) .
$$

For each positive integer $n$, we have

$$
\begin{array}{rl}
\int_{S} g_{n}(t)+\int_{S} & K(s, t) g_{n}(s) d \mu(s) \mid d \mu(t) \\
= & \int_{E_{n}}\left|\frac{1}{\mu\left(E_{n}\right)}+\int_{E_{n}} \frac{1}{\mu\left(E_{n}\right)} K(s, t) d \mu(s)\right| d \mu(t) \\
& +\int_{S \backslash E_{n}}\left|\int_{E_{n}} \frac{1}{\mu\left(E_{n}\right)} K(s, t) d \mu(s)\right| d \mu(t) \\
\geqslant & \int_{E_{n}} \frac{1}{\mu\left(E_{n}\right)} d \mu(t)-\int_{E_{n}}\left|\int_{E_{n}} \frac{1}{\mu\left(E_{n}\right)} K(s, t) d \mu(s)\right| d \mu(t) \\
& +\int_{S \backslash E_{n}}\left|\int_{E_{n}} \frac{1}{\mu\left(E_{n}\right)} K(s, t) d \mu(s)\right| d \mu(t) \\
= & 1+\int_{S}\left|\int_{E_{n}} \frac{K(s, t)}{\mu\left(E_{n}\right)} d \mu(s)\right| d \mu(t)-2 \int_{E_{n}}\left|\int_{E_{n}} \frac{K(s, t)}{\mu\left(E_{n}\right)} d \mu(s)\right| d \mu(t) .
\end{array}
$$

By our choice of $s_{0}$ we have

$$
\lim _{n \rightarrow \infty} \frac{1}{\mu\left(E_{n}\right)} \int_{E_{n}} K_{s} d \mu(s)=K_{s_{0}}
$$

in norm so that

$$
\lim _{n \rightarrow \infty} \int_{S}\left|\int_{E_{n}} \frac{K(s, t)}{\mu\left(E_{n}\right)} d \mu(s)\right| d \mu(t)=\int_{S}\left|K\left(s_{0}, t\right)\right| d \mu(t) \geqslant\|T\|-\varepsilon .
$$


It remains to be shown that

$$
\int_{E_{n}}\left|\int_{E_{n}} \frac{K(s, t)}{\mu\left(E_{n}\right)} d \mu(s)\right| d \mu(t)
$$

can be made arbitrarily small.

Let $E$ be the set of measure 0 and $\mathscr{K}$ be the norm compact subset of $L^{1}(S, \Sigma, \mu)$ for which $K_{s} \in \mathscr{K}$ for all $s \notin E$ as described at the beginning of the proof. Let $\mathcal{K}_{0}=$ norm closure of $\left\{K_{s} \mid s \notin E\right\}$ in $L^{1}(S, \Sigma, \mu)$. Then $\mathcal{K}_{0}$ is compact in the norm topology.

Again consider $\left\{E_{n}\right\}=\left\{E_{n}\left(s_{0}\right)\right\}$. For each positive integer $n$, define $\Phi_{n}$ on $\mathcal{K}_{0}$ by $\Phi_{n}(f)=\int_{E_{n}}|f| d \mu=\int_{E_{n}}|f(t)| d \mu(t)$ for $f \in \mathscr{K}_{0}$. Since $\Phi_{n}$ is a real valued continuous function on the compact set $\mathcal{K}_{0}, \Phi_{n}$ attains a maximum $\gamma_{n}$, say where $\gamma_{n} \geqslant 0$. That is, $\gamma_{n}=\max _{f \in \mathscr{K}_{0}} \int_{E_{n}}|f| d \mu$. Further, since $E_{n} \supset E_{n+1}$, we have $\gamma_{n} \geqslant \gamma_{n+1}$. Let $\gamma=$ $\lim _{n \rightarrow \infty} \gamma_{n}$. We want to show that $\gamma=0$. If $\gamma_{n}=0$ for some integer $n$ then $\gamma=0$. So assume $\gamma_{n}>0$ for all $n$ and let $\mathcal{K}_{n}=$ norm closure of

$$
\left\{K_{s} \mid s \notin E \text { and } \int_{E_{n}}\left|K_{s}(t)\right| d \mu(t)>\frac{\gamma}{2}\right\} .
$$

Since $\mathcal{K}_{n} \subset \mathcal{K}_{0}$ each $\mathcal{K}_{n}$ in compact in the norm topology of $L^{1}(S, \Sigma, \mu)$. Also $\mathscr{K}_{n+1} \subset \mathscr{K}_{n}$ since $E_{n+1} \subset E_{n}$. Further each $\mathcal{K}_{n}$ is nonempty. Therefore $\left\{\mathscr{K}_{n}\right\}$ is a nested sequence of nonempty compact subsets of $L^{1}(S, \Sigma, \mu)$ whence $-\mathcal{K}_{n} \neq \varnothing$. Let $F \in-\mathcal{K}_{n}$. Then $\int_{E_{n}}|F(t)| d \mu(t) \geqslant \gamma / 2$ for all $n$. But $\lim _{n \rightarrow \infty} \mu\left(E_{n}\right)=0$. Therefore $\gamma=0$ as claimed.

Now $\left\{K_{s} \mid s \notin E\right\}$ is dense in $\mathscr{K}_{0}$ so that

$$
\gamma_{n}=\sup _{s \notin E} \int_{E_{n}}|K(s, t)| d \mu(t) .
$$

Since $\gamma_{n} \downarrow 0$ it follows that

$$
\sup _{s \notin E} \int_{E_{n}}|K(s, t)| d \mu(t)<\varepsilon
$$

for large $n$, and so $\int_{E_{n}}|K(s, t)| d \mu(t)<\varepsilon$ for large $n$ and all $s \notin E$. Therefore

$$
\begin{aligned}
\int_{E_{n}}\left|\int_{E_{n}} \frac{K(s, t)}{\mu\left(E_{n}\right)} d \mu(s)\right| d \mu(t) & \leqslant \int_{E_{n}}\left[\int_{E_{n} \backslash E} \frac{|K(s, t)|}{\mu\left(E_{n}\right)} d \mu(s)\right] d \mu(t) \\
& =\int_{E_{n} \backslash E}\left[\int_{E_{n}} \frac{|K(s, t)|}{\mu\left(E_{n}\right)} d \mu(t)\right] d \mu(s) \\
& <\int_{E_{n} \backslash E} \frac{\varepsilon}{\mu\left(E_{n}\right)} d \mu(s) \text { for large } n
\end{aligned}
$$

the equality holding by Tonelli's Theorem. Thus

$$
\int_{E_{n}}\left|\int_{E_{n}} \frac{K(s, t)}{\mu\left(E_{n}\right)} d \mu(s)\right| d \mu(t)<\frac{\varepsilon \mu\left(E_{n} \backslash E\right)}{\mu\left(E_{n}\right)}=\varepsilon
$$


for large $n$. Combining this with $(*)$ we have

$$
\begin{gathered}
1+\int_{S}\left|\int_{E_{n}} \frac{K(s, t)}{\mu\left(E_{n}\right)} d \mu(s)\right| d \mu(t)-2 \int_{E_{n}}\left|\int_{E_{n}} \frac{K(s, t)}{\mu\left(E_{n}\right)} d \mu(s)\right| d \mu(t) \\
>1+\|T\|-\varepsilon-2 \varepsilon=1+\|T\|-3 \varepsilon
\end{gathered}
$$

for large $n$. Since $\varepsilon$ is an arbitrary positive number and $\left\|(I+T) g_{n}\right\|>$ left-hand side of $(* *)$ for each $n$, we have $\|I+T\| \geqslant 1+\|T\|$. Finally, since $\|I+T\| \leqslant 1+\|T\|$ always, we conclude that if $T$ is a compact linear operator on $L^{1}(S, \Sigma, \mu)$ where $(S, \Sigma, \mu)$ is a positive $\gamma$-finite measure space which satisfies Property (V) and $\mu\left(\left\{s_{0}\right\}\right)=0$ for all $s_{0} \in S$, then $\|I+T\|=1+\|T\|$ as required.

3. We conclude with a few general remarks.

(a) If $B$ is finite dimensional, then there exists a compact operator $T$ for which $\|I+T\|<1+\|T\|$. Indeed, every linear operator on $B$ is compact and choosing $T=-I$ gives the required example.

(b) If $B$ is Hilbert space and $\left\{e_{n}\right\}$ is an orthonormal basis then $T: \sum_{n=1}^{\infty} a_{n} e_{n} \rightarrow-a_{1} e_{1}$ is a compact operator for which $\|I+T\|=1<1+\|T\|$.

(c) If $\|I+S\|=1+\|S\|$ holds for every compact operator $S$ in the dual space $B^{*}$ of a Banach space $B$, then $\|I+T\|=1+\|T\|$ for every compact operator in $B$. Indeed, if $T$ is a compact operator on $B$, then $T^{*}$ is compact on $B^{*}$. But $\left\|I^{*}+T^{*}\right\|$ $=1+\left\|T^{*}\right\|$. Hence $\|I+T\|=\left\|I^{*}+T^{*}\right\|=1+\left\|T^{*}\right\|=1+\|T\|$ as claimed.

(d) However, even if $\|I+T\|=1+\|T\|$ for all compact operators $T$ on a Banach space $B$, this property may fail for $B^{*}$. As an example let $B=C[0,1]$. Then $B^{*}=B V[0,1]$. Then $B^{*}=B V[0,1]$. Then $\|I+T\|=1+\|T\|$ for all compact $T$ on $B$; but if we define $S$ on $B V[0,1]$ by $S=-j_{0}(\mu) \nu_{0}$ where $j_{0}(\mu)$ is the jump of $\mu$ at 0 and $\nu_{0}$ is evaluation at 0 , i.e. $\int_{0}^{1} f(t) d \nu_{0}(t)=f(0)$ for all $f \in C[0,1]$. Then using the by now usual argument, $\|I+S\|=1<1+\|S\|$.

\section{BIBLIOGRAPHY}

1. V. F. Babinko and S. A. Picugov, On a property of compact operators on the space of integrable functions, Ukrain Mat. Z. 33 (1981), 491-492.

2. I. K. Daugavet, A property of completely continuous functions on the space $C$, Uspehi Mat. Nauk 18 (1963), 157-158.

3. N. Dunford and J. T. Schwartz, Linear operators. Part I, Interscience, New York, 1958.

4. C. A. Hayes and C. Y. Pauc, Derivation and martingales, Springer-Verlag, Berlin and New York, 1970.

5. G. E. Shilov and B. L. Gurevich, Integral, measure and derivation: a unified approach (translated by R. A. Silverman), Prentice-Hall, Englewood Cliffs, N.J., 1966.

Department of Mathematics, University of Massachusetts at Boston, Dorchester, MasSACHUSETTS 02125 